\title{
A Rare Case of Burkitt Lymphoma in a 13-year-girl
}

\author{
(1) Büşra Yılmaz1', (1) Efsun Somay2, (1) Bermal Hasbay3 \\ 1Başkent University, Adana Dr. Turgut Noyan Research and Practice Center, Department of Dentomaxillofacial Radiology, Adana, Turkey \\ 2Başkent University, Adana Dr. Turgut Noyan Research and Practice Center, Department of Dentomaxillofacial Surgery, Adana, Turkey \\ ${ }^{3}$ Başkent University, Adana Dr. Turgut Noyan Research and Practice Center, Department of Pathology, Adana, Turkey
}

\section{ABSTRACT}

Burkitt lymphoma is rare Non-Hodgkin's lymphoma type in childhood. The differential diagnosis includes dental infection and osteomyelitis. Therefore, patients often refer to the dentist first. Early diagnosis has great importance on prognosis. We aimed to broaden the perspective of dentists about this disease and confirm the diagnosis radiographically and clinically.

Keywords: Burkitt lymphoma, dental abscess, jaw, paediatric

\section{Introduction}

Burkitt lymphoma (BL) first described by Dr. Dennis Burkitt in 1958 (1). It is a sub-type of Non-Hodgkin's lymphoma, and is also an aggressive neoplasm with three variants that are endemic (African), sporadic (American), and immunodeficiency associated [human immunodeficiency virus (HIV) associated] (2).

Although the histopathological features of these three forms are similar, their clinical features are different in terms of age distribution and prevalence. The endemic form that is frequently found in Africa is widely reported to occur in early childhood at a peak age of 6 years; in contrast, the sporadic form does not appear in a particular geographic area and occurs mainly in children and adolescents. The endemic form commonly involves the jaw bone, especially the maxilla, and typically presents as tooth mobility, jaw expansion, and also systemic signs such as an abdominal mass (2). However, jaw involvement in the sporadic form is not common (3).
The HIV associated subtype represents $35-40 \%$ of Non-Hodgkin's lymphomas appearing in the HIV positive population. BL is silent in the early stages, but it grows and expands rapidly. Signs and symptoms vary depending on the primary location and extent of the tumour (4).

Intraoral findings are rarely observed at the beginning of the disease. To emphasize the role of the dentist in the early detection of BL due to the importance of early diagnosis of this rare disease, we present a case of BL in a 13-year-old girl with hypermobile teeth, pain, and numbness on her jaw.

\section{Case Report}

This case report was approved by Başkent University Institutional Review Board (D-KA 20/24) on the $12^{\text {th }}$ February 2020. Informed consent was obtained from the parent of the participant. The authors certify that they have obtained the patient consent form. In the form, the patient has given her consent for her radiological images and other clinical information to be reported in the journal. The patient

\section{Address for Correspondence}

Büşra Yılmaz, Başkent University, Adana Dr. Turgut Noyan Research and Practice Center, Department of Dentomaxillofacial Radiology, Adana, Turkey Phone: +90 5376500773 E-mail: dtbusrayilmaz@gmail.com ORCID: orcid.org/0000-0003-0633-5648

Received: 10.04.2020 Accepted: 21.05.2020

${ }^{\oplus}$ Copyright 2021 by Ege University Faculty of Medicine, Department of Pediatrics and Ege Children's Foundation The Journal of Pediatric Research, published by Calenos Publishing House. 
(parent) understands that her name or initials will not be published and due efforts will be made to conceal her identity, but anonymity cannot be guaranteed.

A 13-year-old girl applied to the Dental Clinic of Başkent University Adana Dr. Turgut Noyan Research and Practice Center with severe dental infections presenting with mandibular and maxillary hypermobile teeth, pain, and numbness of right mandibular region. The patient had been treated for 2 months in another clinic with the diagnosis of dental abscess, receiving intensive antibiotic treatment, and abscess drainage was performed several times by a dentist. These treatments provided temporary relief but symptoms reappeared in a short time.

The patient, whose past medical history was nonspecific, had no history of progressive systemic symptoms such as fatigue, weakness, or fever. Extraoral examination revealed facial asymmetry caused by a soft, painless swelling of the right cheek. Some enlarged lymph nodes were observed. Intraoral examination showed symptoms such as juvenile periodontitis, enlarged gingival mucosa, and several teeth caries. The molar and premolar teeth in both right jaws were mobile and swelling was also observed in the gingiva surrounding these teeth. Purulent pus was not detected. There was no oral sign of disease in the buccal and palate mucosa, upper and lower lips.

In radiological examination, several root resorptions, and chronic periapical infections were determined. On panoramic radiograph, the patient was found to have alveolar bone resorption adjacent to the maxillary and mandibular canines, premolars, and molars with the appearance of floating teeth. Periapical X-rays also attracted attention to the radiological appearance such as a punch hole on the maxillary alveolar bone (Figure 1).

An incisional biopsy was performed on the mandibular molar gingiva. Histopathological findings of the gingiva showed infiltration consisting of small to medium round hyperchromatic vesicular nucleus. Narrow eosinophilic cytoplasm atypical cells were observed under the epidermis in the prepared sections (Figure 2).

Immunohistochemical staining was positive for CD10, CD20, and MUM1, which are specific to B-cell lymphocytes and late stages of $\mathrm{B}$-cell differentiation. It was negative for CD2, CD3, Tdt, MPO, Cyclin D1, CD34, CMYC. Ki-67 staining was positive in more than $85-90 \%$ of the cells, revealing high proliferative activity. Overall, the histopathology results supported the diagnosis of BL. Also, there was no Epstein-Barr virus (EBV) genome (EBV-).

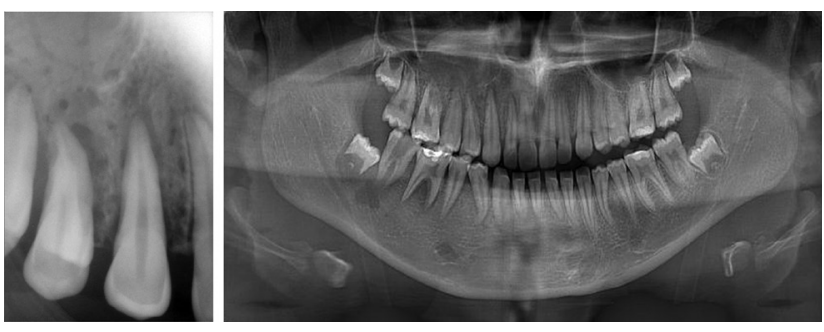

Figure 1. Periapical radiographs have punch holes on the maxillary alveolar bone; panoramic radiograph has alveolar bone resorption and floating tooth appearance from right maxillary and mandibular canines to molars

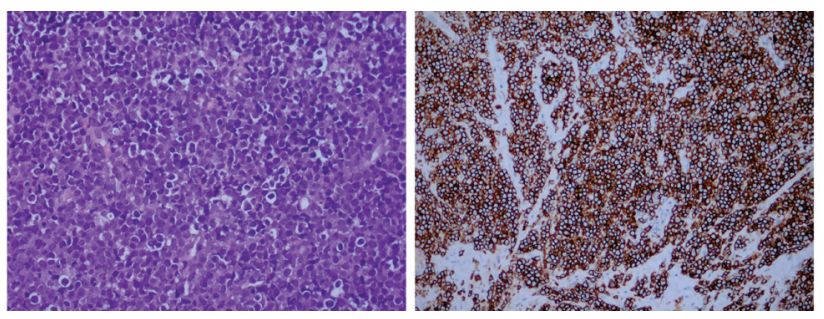

Figure 2. Histopathological evaluation of the patients mandibular molar gingiva

On abdominal examination, a mass in the midline of the pelvis extending to the umbilicus was revealed by the paediatrician. Additionally, nodular masses and accompanying fluid with multiple diffusion restriction in the proximal part of the pancreas corpus, behind the uterus in the pelvic region in the pericecal area and the largest behind the uterus in both ovarian loges were detected via lower abdomen magnetic resonance imaging.

After our prompt biopsy, the extent of the disease was identified, and the patient was immediately referred to the Haematology-Oncology Department for definitive care and chemotherapy was initiated.

\section{Discussion}

Most often affecting the paediatric population, $\mathrm{BL}$ is a potentially curable aggressive neoplasm (5).

Dental radiography can play an important role in the diagnosis of BL. Radiographic changes in the jaw are usually found before the clinical dental signs of the disease appear and can be noticed primarily by dentists (5). In this present case, floating teeth, chronic gingival abscesses, root resorptions of the mandibular posterior teeth, loss of lamina dura, pulpal infiltration with tumour cells, and osteolytic lesions were the problems detected before other symptoms appeared, in accordance with the literature $(6,7)$. Thus, the presence of floating teeth on plain radiography reflecting the destructive process (punch hole appearance) 
in the maxilla and mandible might suggest sporadic BL as well as infectious, hematologic, or metabolic causes including Langerhans cell histiocytosis (7). Moreover, the unexplained hypermobility of teeth and the displacement of developing teeth with cortical weakening in a child were signs that created suspicion regarding malignant lesions (2).

BL should be diagnosed as quickly as possible and requires prompt intervention because it is a rapidly growing high-grade malignant neoplasm (2). However, the clinical features of oral lymphoma are not specific enough for dentists to diagnose, and there is an attempt to solve the misdiagnosed problem by dental treatment. For example, dentists often evaluate hypermobile teeth with a history of jaw pain or floating teeth with resorption of the adjacent alveolar bone as a dental abscess or Langerhans cell histiocytosis, and the prognosis may worsen because the correct diagnosis is delayed. Therefore, dentists should be more attentive in the presence of alveolar bone resorption and/or floating tooth symptoms.

$\mathrm{BL}$ is an aggressive neoplasm with poor prognosis. However, its treatment success depends on its stage. A study on oral BL reported that even stage III patients had a $97 \%$ survival rate. In contrast, the prognosis of stage IV BL is extremely poor: the survival rate of stage IV patients is substantially lower at $27 \%$, suggesting that early diagnosis of $B L$ is crucial (8). Luckily, since the disease was diagnosed early in our case, most organs were not yet involved and the disease did not progress clinically.

Oral signs of $B L$ include the loosening and extrusion of molar teeth, the premature shedding of primary molars and the premature eruption of permanent molars, gingival enlargement and swelling of the alveolar regions and jaw (5). In our case, almost all of these symptoms were observed in accordance with the literature. However, the sporadic type of $\mathrm{BL}$ can involve one quadrant of the jaw, while the endemic type can involve all four quadrants. In this case, the patient had involvement of two quadrants; the right lower and upper jaws. This clinical finding suggests that the BL type in the patient may be an endemic form but it had not progressed to other regions of the jaw as it was diagnosed early.

Since the differential diagnosis of $\mathrm{BL}$ is acute dental infection and osteomyelitis (5), when these are not supported radiologically, these findings may lead the dentist to make a wrong diagnosis, as in our case. In such a situation, the patient's disease may have progressed beyond treatment and be fatal. In our case, the patient had been diagnosed with dental abscess and was given intensive antibiotic treatment and abscess drainage was carried out twice over a period of two months, and the real diagnosis of the disease was delayed because no detailed radiographic examination was performed.

According to the literature, the disease does not show systemic symptoms such as weakness, fever, hepatosplenomegaly, and is diagnosed in children only with intraoral and extraoral findings. It has been reported that the loss of lamina dura is noticeable radiologically. In our case, there were no systemic findings and the disease was diagnosed completely via oral findings. Notably, the presence of punch hole in the alveolar bone on periapical radiographs distinguishes this case from others $(7,9)$.

Furthermore, in a case of BL in 15-year-old girl, attention was drawn to misdiagnosis of this disease. In that case report, the dentist tried to treat the swelling of the patient in the maxillary area with root canal treatment, initially considering it to be endodontic lesion as in our case (9).

In addition to the facial mass in the sporadic form, toothache and paresthesia on the lip are frequently seen (5). Similar to the literature, our patient had had toothache and paraesthesia in the right lower lip for 3 months.

The sporadic form of BL is more common in boys than in girls and is most commonly seen between the ages of 3-8. The endemic form occurs in both genders and the average age is 11 . In our case, the age of the patient was 13 and the gender was female, making us think that the diagnosis was more suitable for the endemic form (2). EBV appears in $97 \%$ of endemic LB, but only in $20-30 \%$ of sporadic cases (10). The EBV genome was not present in our case.

Abdominal mass is frequently seen in older children and adults in the sporadic form of BL (11). The patient we present in this paper has a mass in the midline of the pelvis extending to the umbilicus.

\section{Conclusion}

This case suggests the possibility of dental complaints as an initial clinical manifestation of $\mathrm{BL}$ and emphasizes the role of dentists in the early detection of this disease to improve prognosis, as early diagnosis and immediate chemotherapy treatment are important in increasing the survival rate.

\section{Ethics}

Informed Consent: Informed consent was obtained from the parent of the participant.

Peer-review: Externally and internally peer-reviewed. 


\section{Authorship Contributions}

Concept: B.Y., E.S., B.H., Design: B.Y., E.S., B.H., Data Collection or Processing: B.Y., E.S., B.H., Analysis or Interpretation: B.Y., E.S., B.H., Literature Search: B.Y., E.S., B.H., Writing: B.Y., E.S., B.H.

Conflict of Interest: No conflict of interest was declared by the authors.

Financial Disclosure: The authors declared that this study received no financial support.

\section{References}

1. Ajila V, Gopakumar R, Hegde S, Babu SC. Intraoral Burkitt's lymphoma in an HIV positive patient. Indian I Sex Transm Dis AIDS 2012; 33:118-20.

2. Ferry JA. Burkitt's lymphoma: clinicopathologic features and differential diagnosis. Oncologist 2006; 11:375-83.

3. Lee DH, Yu MS, Lee BJ. Primary burkitt's lymphoma in the nasal cavity and paranasal sinuses. Clin Exp Otorhinolaryngol 2013; 6:184-6.

4. Shapira J, Peylan-Ramu N. Burkitt's lymphoma. Oral Oncol 1998; 34:15-23.
5. Maringonda Vicente KG, Ito FA, de Lima HG, Tanaka EE, Takahashi R, Takahama A. Widespread sporadic type burkitt lymphoma presenting as dental mobility and premature eruption of permanent teeth in a child. ASDC I Dent Child 2020; 87:53-7.

6. Martinelli-Klay CP, Martinelli CR, Martinelli C, Dias JB, Cheade TC, Lombardi T. Primary extranodal non-Hodgkin lymphoma of the gingiva initially misdiagnosed as dental abcess. Quintessence Int 2009; 40:805-8.

7. $\mathrm{Cho}, \mathrm{BH}$, Shin $\mathrm{DH}$, Jung $\mathrm{YH}$, Park HR. Widely disseminated sporadic Burkitt lymphoma initially presented as oral manifestations in a 6-year-old boy. I Oral Biol Craniofac Res 2018; 8:140-2.

8. Otmani N, Khattab M. Oral burkitt's lymphoma in children: the Moroccan experience. Int J Oral Maxillofac Surg 2008; 37:36-40.

9. Cabras M, Arduino PG, Chiusa L, Broccoletti R, Carbone M. Case report: sporadic Burkitt lymphoma misdiagnosed as dental abcess in a 15-year-old girl. F1000Res 2018; 7:1567.

10. Banthia $V$, Jen $A$, Kacker $A$. Sporadic Burkitt's lymphoma of the head and neck in the pediatric population. Int I Pediatr Otorhinolaryngol 2003; 67:59-65.

11. Valenzuela-Salas B, Dean-Ferrer A, Alamillos-Granados F). Burkitt's lymphoma: a child's case presenting in the maxilla. Clinical and radiological aspects. Med Oral Patol Oral Cir Bucal 2010;15:e479-82. doi: 10.4317/medoral.15.e479. 